\title{
Decreased expression levels of Ifi genes is associated to the increased resistance to spontaneous arthritis disease in mice deficiency of IL-1RA
}

Xiaoyun Liu ${ }^{1,2+}$, Yan Jiao ${ }^{1,2^{*}+}$, Yanhong Cao ${ }^{2,4,5}$, Nan Deng ${ }^{6}$, Yonghui Ma ${ }^{2}$, Karen A. Hasty ${ }^{2,7}$, Andrew Kang ${ }^{6}$, Hong Chen ${ }^{3}$, John M. Stuart ${ }^{6,7}$ and Weikuan $\mathrm{Gu}^{2,7^{*}}$

\begin{abstract}
Background: The mouse strain BALB/C deficient in IL-1 receptor antagonist protein (II-1 ra) develops spontaneous arthritis disease (SAD) while the strain DBA $/ 1 / \mathrm{Lrn}^{-1-}$ with the same deficiency does not. Previously, we mapped a QTL on chromosome 1 for SAD and then developed a congenic mouse strain BALB.D1-1 $1^{-1-}$ that contains the QTL genomic fragment associated with resistance from $\mathrm{DBA} / 1^{-1-}$ on a BALB/ $/ \mathrm{c}^{---}$background. The congenic strain was relatively resistant to spontaneous arthritis and had delayed onset and reduced severity of disease. We obtained whole genome expression profiles from the spleen of the congenic strain BALB.D1-1 ${ }^{-1-}$ and four other strains, the wild type BALB/C, DBA/1 and the deficient DBA/1 $/ \mathrm{Lrn}^{-1-}$ and the BALB/C $/ \mathrm{Lr} \mathrm{rn}^{-1-}$. We then compared the similarities and differences between the congenic strain and the four parental strains. Here we report the selected potential causal genes based on differential expression levels as well as function of genes.
\end{abstract}

Results: There is a considerable number of genes that are differentially expressed between the congenic strain and the three parental strains, $\mathrm{BALB} / \mathrm{c}, \mathrm{DBA} / 1$, and $\mathrm{DBA} / 1^{-/-}$. However there only a few differentially expressed genes were identified by comparing the congenic strain and the BALB/ $\mathrm{C}^{-1}$ strain. These differentially expressed genes are mainly from T-cell receptor beta chain (Tcrb) and interferon-activatable protein (Ifi) genes. These genes are also differentially expressed between congenic strain and BALB/C strains. However, their expression levels in the congenic strain are similar to that in DBA/1 and DBA $/ 1^{-1-}$. The expression level of Tcrb-j gene is positively associated with two genes of Ifi gene 200 cluster.

Conclusions: Decreased expression levels of Ifi genes is associated to the increased resistance to spontaneous arthritis disease and with down regulation of expressions of Tcrb genes in the mouse congenic strain. Ifi genes may play an important role in the susceptibility to SAD in mice.

\section{Background}

Identification of genes that regulate susceptibility to arthritis is essential in the selection of molecular targets for therapeutic application. The primary locus regulating collagen induced arthritis (CIA) was found to be within

\footnotetext{
*Correspondence: yjiao2@uthsc.edu; wgu@uthsc.edu

${ }^{\dagger}$ Equal contributors

${ }^{1}$ Mudanjiang Medical College, Mudanjiang, HeilongJiang 157001, People's Republic of China

${ }^{2}$ Department of Orthopaedic Surgery and Biomedical Engineering, University of Tennessee Health Science Center (UTHSC), Memphis, TN 38163, USA Full list of author information is available at the end of the article
}

the major histocompatibility complex (MHC) loci [1-5]. This was expected because it was established that CIA in both rats and mice is MHC-linked [6-10]. Tremendous research has been done on the study of molecular mechanism of CIA model [11-15]. However, a considerable contribution to the arthritis is caused by a non-MHClinked molecular mechanism [16-23]. Many non-MHClinked loci have been identified [19, 22, 23], yet the causal genes for these QTL have not been known. Understanding of genes that regulate susceptibility to non-MHC-linked arthritis is essential for-the selection of molecular targets for therapy for non MHC-linked arthritis. 
$\mathrm{BALB} / \mathrm{c}$ and $\mathrm{DBA} / 1$ are important pairs of mouse strains. BALB/c and DBA/1 and C57B6/J mice share the same MHC (H-2d) haplotype; however, at the whole genome level they are genetically distant $[17,24]$. In the mouse model, with a standard protocol, CIA is induced in DBA/1 and B6 $[3,10,11,25]$. Under the same standard protocol, CIA could not be induced in BALB/c mice. The spontaneous arthritis (SAD) occurs in interleukin-1 (IL-1) receptor antagonist (IL-1rn) -deficient mice, which is dependent on non-MHC genetic bases $[25,26]$.

Over the last decade, we have been studying SAD in IL-1ra-deficient mice [25-29]. To better understand the pathogenesis of SAD, initially we used classical genetic techniques and bred susceptible and resistant mice to obtain an F2 generation and identified QTL associated with arthritis susceptibility [26]. We obtained evidence for potential QTL on chromosomes 1, 6, 11, 12, and 14 . The QTL on the chromosome 1 was the major regulator for the SAD. To confirm the importance of the QTL and to identify potential candidate genes within it, we conducted speed congenic breeding to transfer the QTL region from DBA/1 mice that are resistant to spontaneous arthritis into BALB $/ \mathrm{c}^{-1-}$ which are susceptible [29]. Our congenic breeding was successful in identifying a QTL associated with the development of spontaneous arthritis. When a fragment of DNA from the DBA/1 strain was introduced onto a BALB/c background, arthritis was delayed in onset and was less severe. Using the congenic strains that we developed, the genomic region of the originally identified QTL was redefined into a region that is downstream from the peak region of our original mapping $[26,27,29]$.

Microarrays have been used for the analysis of whole genome expression profiles for the more than a decade. They have developed as a mature technology not only in the producing of gene expression profiles but also in the analysis of differentially expressed genes. One of the useful applications of microarray-generated whole genome expression profiling is the identification of candidate genes that regulate a specific disease trait or a molecular pathway [28, 30-34].

According to the Ensembl database, the transferred region from DBA/1-/- to BALB/c-/- is between D1Mit110 and D1Mit209 on chromosome 1 with a size of $23.73 \mathrm{Mb}$ which contains 320 genetic elements. Among those genes, 115 are identified as genes relevant to arthritis and its potential pathways [29]. Although the number of genes seems large and to some degree difficult to specifically target candidate genes, comparison of the data from congenic strains provide a defined genomic region for their localization. The use of congenic strains enable us to analyze the effect of the transferred fragment within QTL region on global gene expression. Previously, we have been using the microarray tools for the study of differential gene expression comparing diseased knockout and normal wild type mice [28, 32, 33]. In this study, we compared gene expression profiles of the whole genome of the congenic strain and parental strains.

\section{Methods \\ Mice}

Female mice at 4 months of age from a congenic strain BALB.D1-1, BALB/c and DBA/1 wild type, and BALB/c and DBA/1 Il1rn knockout mice (Table 1) [29] were used to generate gene expression data using the Illumina platform. The strain BALB.D1-1 is on the BALB/c genomic background contains genetic DNA from DBA/1 between D1Mit55 and D1MitD1Mit209 inclusive. Analysis of each strain was done with three spleens, each from a different female mouse. All mice have been maintained in the animal facility of the Department of Veterans Affairs Medical Center, Memphis. Experimental animal procedures and mouse husbandry were performed in accordance with the National Institutes of Health's Guide for the Care and Use of Laboratory Animals and approved by the VAMC Institutional Animal Care and Use Committee.

\section{Genotyping}

The insertion and genotype of the congenic BALB.D1-1 mice was confirmed before RNA extraction and microarray analysis [26]. Genomic DNA was extracted from tissues obtained by ear punch. The procedure used has been previously described [20]. Briefly, DNA was extracted from the tissue and amplification of microsatellite markers conducted by polymerase chain reaction (PCR). PCR products were analyzed using poly-acrylamide gel electrophoresis using the Mega-Gel Dual High-Throughput Vertical Electrophoresis System (C.B.S. Scientific, Del Mar, CA).

\section{RNA extraction}

RNA was extracted from spleens using a Trizol reagent (Invitrogen, CA). Total RNAs were purified using the RNeasy MinElute Cleanup Kit (Qiagen, CA). RNA quality and integrity were analyzed by the Agilent Bioanalyzer [33].

\begin{tabular}{|c|c|c|}
\hline Strain & $\begin{array}{l}\text { IL-1 Receptor } \\
\text { Antagonist }\end{array}$ & Description \\
\hline $\mathrm{BALB} / \mathrm{C}$ & $+/+$ & $\begin{array}{l}\text { Normal strain originally from the } \\
\text { Jackson laboratory }\end{array}$ \\
\hline $\mathrm{DBA} / 1$ & $+/+$ & $\begin{array}{l}\text { Normal strain originally from the } \\
\text { Jackson laboratory }\end{array}$ \\
\hline $\mathrm{BALB} / \mathrm{C}^{-/-}$ & $-/-$ & With mutation of $1 L 1 \mathrm{rn}$ \\
\hline $\mathrm{DBA} / 1^{-/-}$ & $-/-$ & With mutation of $1 L 1 \mathrm{rn}$ \\
\hline BALB.D1-1 & $-/-$ & $\begin{array}{l}\text { With mutation of } \| L 1 \mathrm{r} \text {, with a piece } \\
\text { of fragment from } D B A / 1 \text {, with } \\
\text { BALB/c background. }\end{array}$ \\
\hline
\end{tabular}




\section{Microarray procedure}

A starting amount of 200 ng of high-quality total RNA, with a RIN (RNA Integrity Score) number of more than seven, was used to generate cDNA and cRNA using the Illumina ${ }^{\oplus}$ TotalPrep $^{\mathrm{Tm}}$ RNA Amplification Kit (Ambion). For each of five individual samples, 1.5 ug of cRNA sample was hybridized overnight to Illumina mouse- 6 v1.1 expression beadchips in a multiple step procedure according to the manufacturer's instructions; the chips were washed, dried and scanned on the BeadArray Reader (Illumina, CA) and raw data were generated using BeadStudio 2.3.41 (Illumina, CA) [28, 33].

\section{Analysis of microarray data}

Raw data were normalized with quantile methodology using BeadStudio software. Four comparisons were made. The gene expression profiles of BALB.D1-1 compared to that of $\mathrm{BALB} / \mathrm{c}^{-1-}, \mathrm{BALB} / \mathrm{c}$ wild type, $\mathrm{DBA} / 1^{-1-}$, and DBA/1 wild type. The Diff Score from the quantile method is a transformation of the $\mathrm{p}$-value that provides directionality to the p-value based on the difference between the average signal in the reference group vs. the comparison group. For a p-value of 0.05 , DiffScore $= \pm 13$; For a $p$-value of 0.01 , DiffScore $= \pm 22$; For a $p$-value of 0.001 , DiffScore $= \pm 33$. The DiffScore in our initial analysis was set up as \pm 10 , to ensure we will not miss the potential candidate genes $[33,35]$.

\section{Candidate and pathway analysis and construction}

To identify candidate genes and construct pathway/connections, we correlated expression between candidate genes within QTL region and Il1rn, including Il1a, Il1b, Il1r1, Il1r2. For pathway analysis, we took full advantage of existing data of gene expression profiles at GeneNetwork at http://www.genenetwork.org/webqtl/main.py. We used gene expression data from spleens obtained from a set of recombinant inbred (RI) strains derived from C57BL/6 J (B6) and DBA/2 J (D2) [18, 36]. Data was generated using the Affymetrix GeneChip Mouse Gene 1.0 ST array (http://www.genenetwork.org/webqtl/main.py? FormID=sharinginfo\&GN_AccessionId = 283). In the case of multiple probes, the one with highest expression level was chosen for the analysis [37]. The gene networks were constructed using application tools in GeneNetwork. We constructed the gene network based on the Network Graph in combination with the Correlation Matrix. Network graph is often used to visualize multiple sets of interactions. The Spring Model layout (force reduction) was used for the graphic method for all graphic samples. We followed standard criteria for the strong, correlation, correlation, and none correlation. When the $\mathrm{R}$ value is equal or more than 0.7 or -0.7 , we regard the correlation is strong positive or negative correlation. When the $\mathrm{R}$ value is between 0.5 and 0.69 or -0.5 and -0.69 , the correlation exists but not as strong. Any $\mathrm{R}$ value between 0 and 0.5 or 0 and -0.5 is treated as none-to-weak-correlation [33, 35]. Initial analysis of relevance of genes to arthritis was conducted using PGMapper [37].

\section{Results \\ Similarities of whole genome gene expression profiles among different strains}

The congenic strain BALB.D1-1 contains a genomic fragment flanked by markers D1Mit55 and D1Mit209 from DBA/1 in BALB/c background [20]. We first examined the similarities of whole genome gene expression of BALB.D1-1 to the other four strains of mice. The Illumina mouse-6 v1.1 expression beadchip contains 30774 probes (46632 elements) representing 29940 mouse genes. We calculated the $\mathrm{R}$ (Correlation) of expression levels of probes between BALB.D1-1 and the other four strains (Fig. 1). Although $\mathrm{R}$ values to those four strains indicates that the expression profiles of BALB.D1-1 is significantly related to all of those four strains, there are slight differences among them. Because the BALB.D1-1 is deficient in IL-1 receptor antagonist protein (IL-1RA), its expression profile is more similar to the two knockout strains, ${ }^{-/-} \mathrm{DBA} / 1^{-/}$and $\mathrm{BALB} / \mathrm{c}^{-/-}$, than to the wild type strains, $\mathrm{BALB} / \mathrm{c}$ and DBA/1. Because of the insertion of a fragment from DBA/1, BALB.D1-1 acts similar to ${ }^{-/-} \mathrm{DBA} / 1^{-/-}$, showing resistance to spontaneous arthritis [20] with a gene expression profile resembling the ${ }^{-/} \mathrm{DBA} / 1^{-/}$. However, because BALB.D1-1 is on the BALB/c background, it is least related to DBA/1 with the $\mathrm{R}$ value of 0.976964 .

\section{Gene expression profiling between BALB.D1-1 and DBA/1 wild type}

Because the BALB.D1-1 is under the BALB/c background with the spall piece of fragment from DBA background, one would expected to see the similarity of a small number of genes in the expression levels. However, the expression levels of more than $97 \%$ of genes between these two strains are similar. We then focused on the genes in the QTL region. We found that all of the genes in this region are expressed at the similar level between these two strains." In particular, we noticed that all of the genes in the interferon activated (Ifi) gene family are similar between these two strains.

Compared to the expression levels of DBA/1, the expression levels of 264 probes were down regulated and 472 probes were up regulated in the BALB.D1-1 (Fig. 2a, b). Among the 472 up regulated probes, 344 are known genes. By searching the PubMed using PGMapper, we found that, among the 344 known genes, 61 are arthritis relevant. Two genes represented by those 472 probes are located in the QTL region, Darc and Fcgr4 (Additional file 1: Table S1). Among the 264 down regulated probes, three are within the QTL region, Kmo, Fcrla, and Ephx1. 


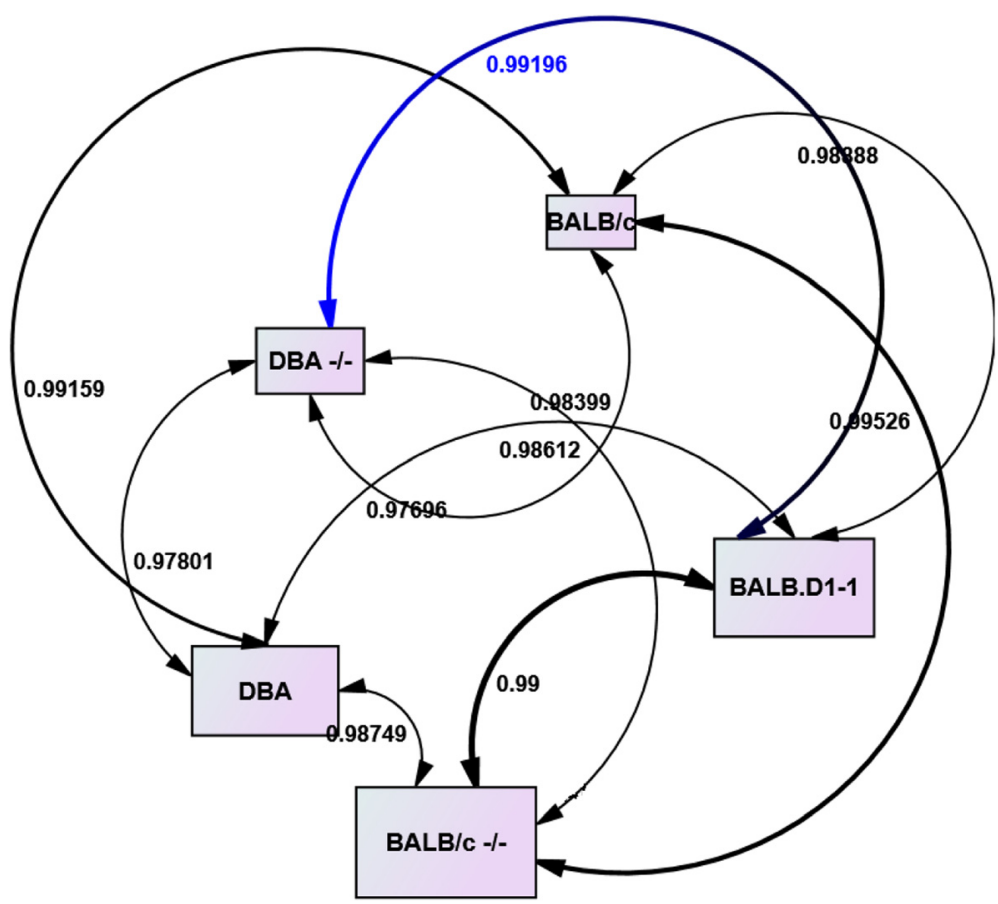

Fig. 1 Correlation between among BALB.D1-1 and four strains. Strain names are listed on the left vertical bar. R values are listed under the horizontal bar. The gene expression profile of wild type DBA/1 (DBA) has the lowest similarity with that of congenic mice, which has a mutation of $\| 1 \mathrm{rn}$ and is on the BALB/c genomic background.in the square boxes. Double-arrow lines are used to connect the two strains in comparisons. Correlation $R$ values are listed along the arrow lines

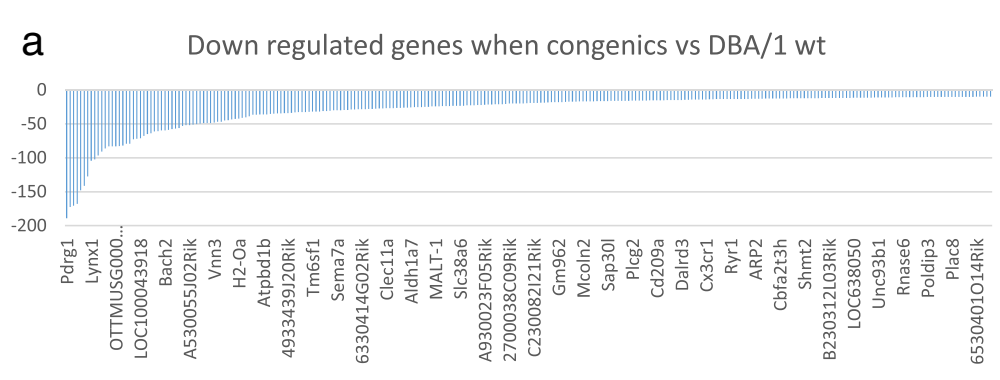

b Up regulated genes when congenics vs DBA/1 wt

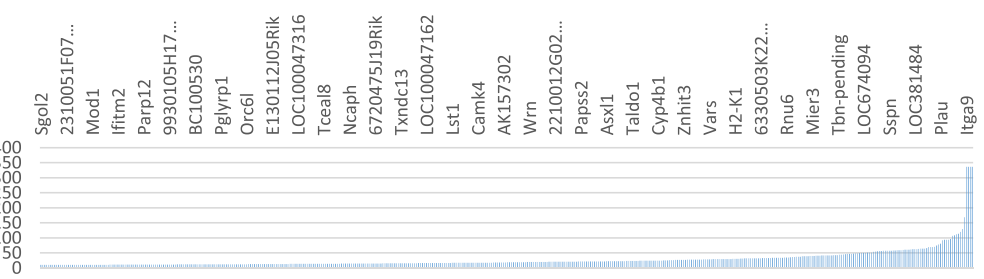

Fig. 2 Up and down regulated genes and networks in congenic mice in comparison to that of DBA wild type mice. a Down regulated genes in congenic mice. Probe/gene names are shown above the horizontal bar. Numbers on the left of vertical bar is the score of differential expression. b Up regulated genes in congenic mice. Cut off score as significant is -2 or 2. Probe/gene names are shown above the horizontal bar. Numbers on the left of vertical bar is the score of differential expression 
Probes of 189 out of 264 represent known genes. Among those known genes, 26 are categorized by PGMapper as arthritis relevant genes (Additional file 1: Table S1).

The 61 up regulated and 26 down regulated genes together with Il1a, Il1b, Il1r1, Il1r2, Il1rap, Il1rapl1, Il1rn are analyzed at Genenetwork. Using UTHSC Affy MoGene 1.0 ST Spleen (http://www.genenetwork.org/webqtl/main. py?FormID=sharinginfo\&GN_AccessionId=283) were identified 162 probes. After eliminating some duplicate probes, 138 probes were used for the gene network construction [38]. The gene Network shows that none of these genes directly connect to the Il1rn or other interleukin 1 family genes (Additional file 2: Figure S1).

Thus, none of the genes that showed differential expression are likely candidate genes that lead to the increased resistance to SAD.

\section{Gene expression profiling between BALB.D1-1 and -/DBA $/ 1^{-/-}$}

Both BALB.D1-1 and ${ }^{-/}$DBA $/ 1^{-/}$are lack of IL1rn gene. Their gene expression levels showed more similarity in comparing to that between BALB.D1-1 and ${ }^{-1-}$ DBA 1 . We confirmed that the expression levels of Ifi gene family are similar between these two strains.

We then conducted further comparison to eliminate the possibility of other genes as favorite candidate genes. Compared to the expression levels of DBA $/ 1^{-1-}$, the expression levels of 241 probes were down regulated and 310 probes were up regulated in the BALB.D1-1 (Fig. 3a, b). Among the 310 up regulated probes, 235 are from known genes (Additional file 3: Table S2). Searching PubMed with
PGMapper on Oct 2, 2013, 43 of those known genes were found to be relevant to arthritis. Among the 241 down regulated probes, 212 are from known genes (Additional file 3: Table S2). Searching PubMed with PGMapper on Oct 2, 2013, 29 of those known genes were found to be relevant to arthritis. A total of 138 probes for the 43 up and 29 down regulated and the seven Il1rn family genes were identified from the Using UTHSC Affy MoGene 1.0 ST Spleen. After eliminating some duplicate probes, 101 probes were used for the analysis [28]. Similar to that of DBA wt mice, data analyses of gene networks indicate that there is no strong connection of expression levels between these genes and Il1rn family. (Additional file 2: Figure S2).

\section{Gene expression profiling between BALB.D1-1 and BALB/C} Compared to the expression levels of $\mathrm{BALB} / \mathrm{c}$, the expression levels of 31 probes were down regulated and 70 probes were up regulated in the BALB.D1-1 (Fig. 4a, b). Among the 70 up regulated probes, all are from known genes. One gene was found in QTL region, Ifi202b. Among 31 down regulated probes, 30 are from known genes. Comparing to genes in QTL region, one gene is in the list of the QTL region, Ifi203 (Additional file 4: Table S3). The 70 up regulated and 30 down regulated genes were analyzed using PGMapper and Siglec1 was found to relevant to arthritis. The 128 probes were identified to represent the 70 and 30 up and down regulated genes respectively and the Ilrn family. After removing the duplicate probes, 92 probes were used for the gene network construction. The gene network indicated that that none of these genes directly connected to the Il1rap

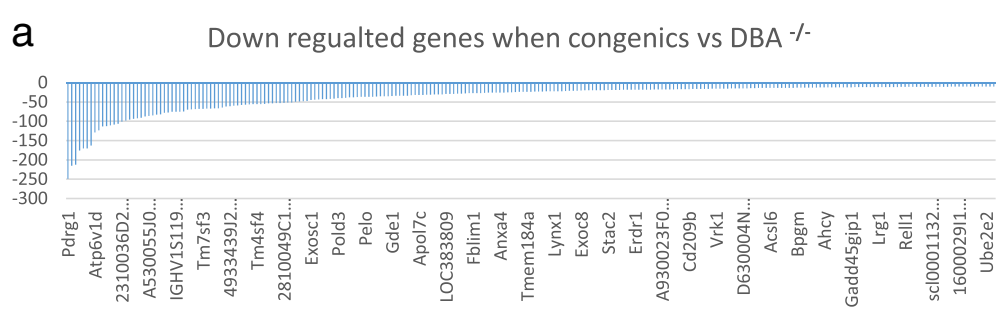

b Up regualted genes when congenics vs DBA $\%$

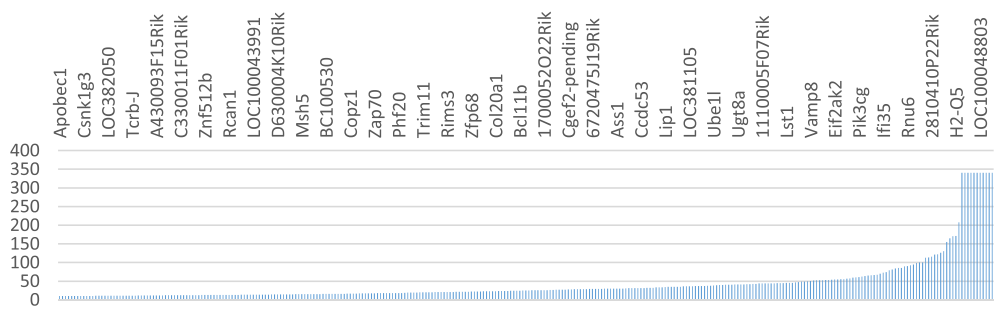

Fig. $3 \mathrm{Up}$ and down regulated genes and networks in congenic mice in comparison to that of ${ }^{-1} \mathrm{DBA} / 1^{-1}$ mice. a Down regulated genes in congenic mice. Probe/gene names are shown above the horizontal bar. Numbers on the left of vertical bar is the score of differential expression. b Up regulated genes in congenic mice. Cut off score as significant is -2 or 2. Probe/gene names are shown above the horizontal bar. Numbers on the left of vertical bar is the score of differential expression 
a Down regualted genes when congenics vs Balb/c

wt

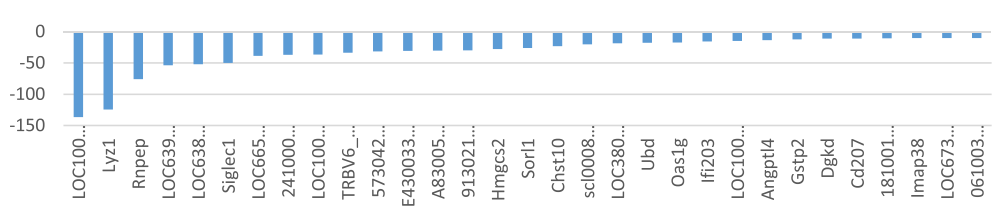

b

Up regualted genes when congenics vs Balb/c wt

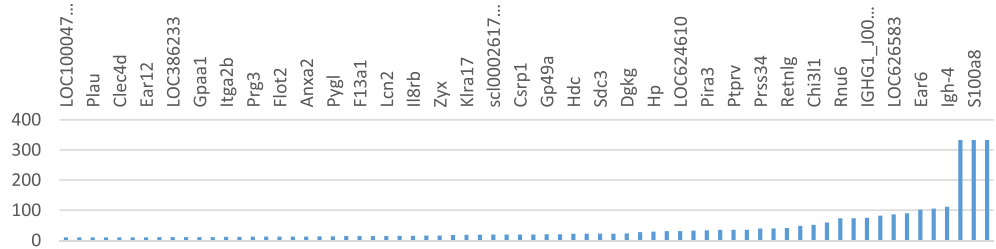

Fig. $4 \mathrm{Up}$ and down regulated genes and networks in congenic mice in comparison to that of BALB/c wild type mice. a Down regulated genes in congenic mice. Probe/gene names are shown above the horizontal bar. Numbers on the left of vertical bar is the score of differential expression. $\mathbf{b}$ Up regulated genes in congenic mice. Cut off score as significant is -2 or 2 . . Probe/gene names are shown above the horizontal bar. Numbers on the left of vertical bar is the score of differential expression

or other interleukin 1 family genes (Additional file 2: Figure S3).

\section{Gene expression profiling between BALB.D1-1 and BALB/ $\mathrm{c}^{-1-}$} Compared to the expression levels of $\mathrm{BALB} / \mathrm{c}^{-/-}$, the expression levels of 12 probes were down regulated, but no probes were significantly up regulated in the BALB.D1-1 (Fig. 5a). Among the 12 down regulated probes, 8 are from the known genes or sequences, LOC100040462 (Mndal), LOC639001 (T-cell receptor beta chain V region C5 precursor), Ifi203, LOC665425 (T-cell receptor beta chain V region LB2 precursor), Lefty1, Trbc6, LOC638301, Ifi204, Ifi202b (Fig. 6a). Based on the search of results from PubMed with PGMapper, none of those known genes were found to be relevant to arthritis. The interferon activated gene family (Ifi202b, Ifi203, Ifi204, Mndal) and Lefty1 are located in QTL region.

Six of the 11 probes (Rnpep, Ifi203, Ifi204, Ifi205, Lefty1, Ifi202b) were found in the UTHSC Affy MoGene 1.0 ST Spleen database. Those 6 probes are analyzed together with Il1a, Il1b, Il1r1, Il1r2, Il1rap, Il1rapl1, Il1rn at Genenetwork. Using the whole genome expression profile from the spleen, we constructed the network of these genes (Fig. 5b), which shows that there is no strong correlation between the expression levels of IL1rn family and the interferon-activatable protein (Ifi) family members. We then constructed the gene network using whole genome expression profiles from the HZI T-Helper Cell Affymetrix M430v2 (http://www.genenetwork.org/webqtl/ main.py?FormID=sharinginfo\&GN_AccessionId=319) and HZI T-regulatory $\mathrm{T}$ cells (CD4+CD25+) (http://www.
genenetwork.org/webqtl/main.py?FormID=sharinginfo\& GN_AccessionId=122). Data for gene networks of both T helper cells and $\mathrm{T}$ regulatory cells support that there is no strong connection between the expression levels of IL1rn family and the Ifi family members (Fig. $5 \mathrm{c}$ and d). Even after a further decrease of the threshold for significance to 0.35 , there still no direct correlation between IL1rn and the If $i$ family members.

Potential involvement of interferon-activatable protein and T-cell receptor beta chain genes in the resistance to SAD

We next compared the expression levels of all the 12 down regulated genes through the comparison between the congenic strain and the four parental strains above. As shown in Fig. 6, 11 of 12 down regulated probes in the congenic strain, compared to $\mathrm{BALB}^{-1}$ mice, were also down regulated when compared to that of $B A L B / c$ wt. None of the genes were down regulated when compared to that of ${ }^{-1} \mathrm{DBA} / 1^{-/}$and DBA/1 wt. These data means that the expression levels of these genes are high in the $\mathrm{BALB} / \mathrm{c}$ and $\mathrm{BALB}^{-/}$while the expression levels of these genes are low in DBA/1 wt and ${ }^{-/-} \mathrm{DBA} / 1^{-/}$. Considering the fact that ${ }^{-1} \mathrm{DBA} / 1^{-/}$is resistant to SAD while $\mathrm{BALB}^{-/-}$ is susceptible to SAD, the decreased expression level of these genes in the congenic stain suggests that the increased resistance in congenic mice may be due to the down regulation of these genes.

Because T-cell receptor beta chain genes are not located on mouse chromosome 1 . They are not the causal genes for the QTL. The only other group of differential expressed genes are the genes of If $i$ cluster. If the genes 


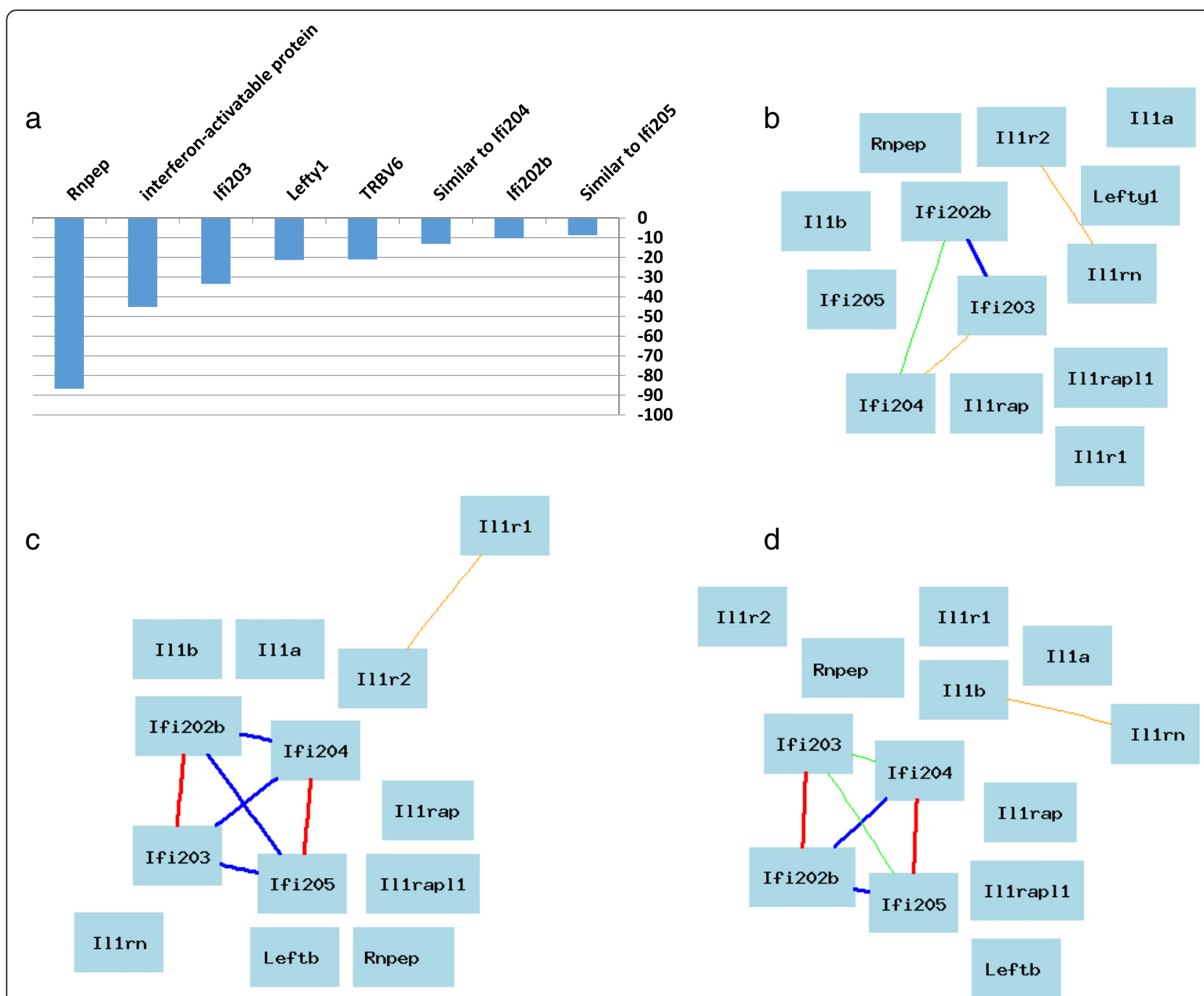

Fig. 5 Down regulated genes and network in congenic mice in comparison to that of BALB/ $\mathrm{c}^{-/}$type. a Down regulated genes in congenic mice. Probe/gene names are shown above the horizontal bar. Numbers on the left of the vertical bar is the score of differential expression. $\mathbf{b}$ Network of known arthritis relevant down regulated genes when congenics are compared to BALB/ $\mathrm{C}^{-1}$ based on gene expression profiles in the spleen. The 13 nodes in the graph below show the selected traits. All nodes are displayed. The 4 edges between the nodes, filtered from the 78 total edges and drawn as curves, show Pearson correlation coefficients greater than 0.5 or less than -0.5 . The graph's canvas is 40.0 by $40.0 \mathrm{~cm}$, and the node labels are drawn with a 16.0 point font, and the edge labels are drawn with a 16.0 point font. c Network of known arthritis relevant down regulated genes when congenics vs BALB/ $\mathrm{c}^{-1}$ based on gene expression profiles in T help cells. The seven edges between the nodes, filtered from the 78 total edges and drawn as curves, show Pearson correlation coefficients greater than 0.5 or less than -0.5 . $\mathbf{d}$ Network of known arthritis relevant down regulated genes when congenics are compared to BALB/ $\mathrm{C}^{-1}$-based on gene expression profiles in T cell regulatory cells

of Ifi200 cluster is the causal genes for the QTL, then the decreased expression level of T-cell receptor beta chain genes should be caused by the decreased level of genes of Ifi200 cluster. Thus, there should be positive correlation at gene expression levels between at least one T-cell receptor beta chain gene and at least one gene in the If 200 cluster. We then identified probes for Ifi 200 cluster and one probe for T-cell receptor beta, joining region (Tcrb-j) from the whole genome profiles of mouse spleen in BXD strains. Our analysis shows that the expression levels of Ifi203 and Ifi205 are positively associated to that of Tcrv-j (Fig. 6b and c). Thus, in the case of congenic strain, the decreased expression levels of Ifi203 and Ifi205 may lead to the decreased expression levels of $\mathrm{T}$-cell receptor beta chain genes. In turn, the down regulation of expression levels of T-cell receptor beta chain genes may lead to the increased resistance to spontaneous arthritis disease in the mouse congenic strain.

\section{Real-time PCR for confirmation of expression level of Ifi203}

Although our laboratory has been conducting microarray analysis for the past 13 years and we have eliminated all 
a

b

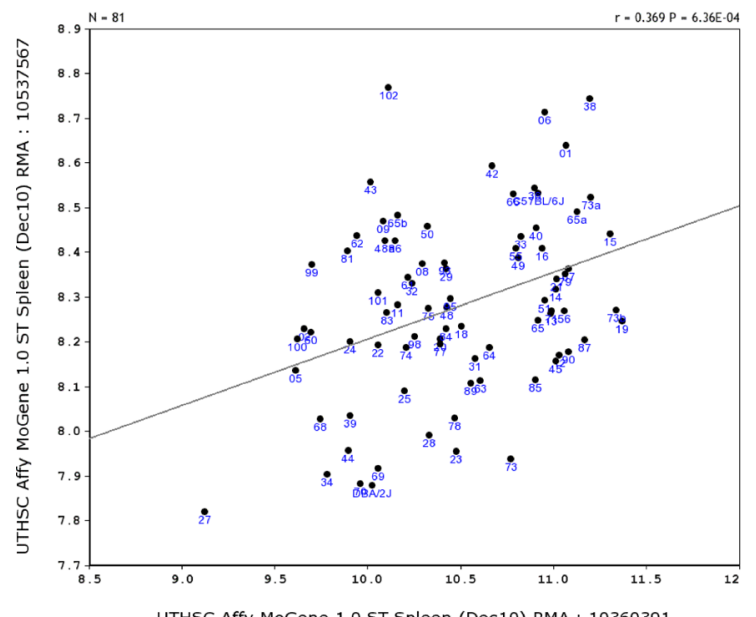

$c$

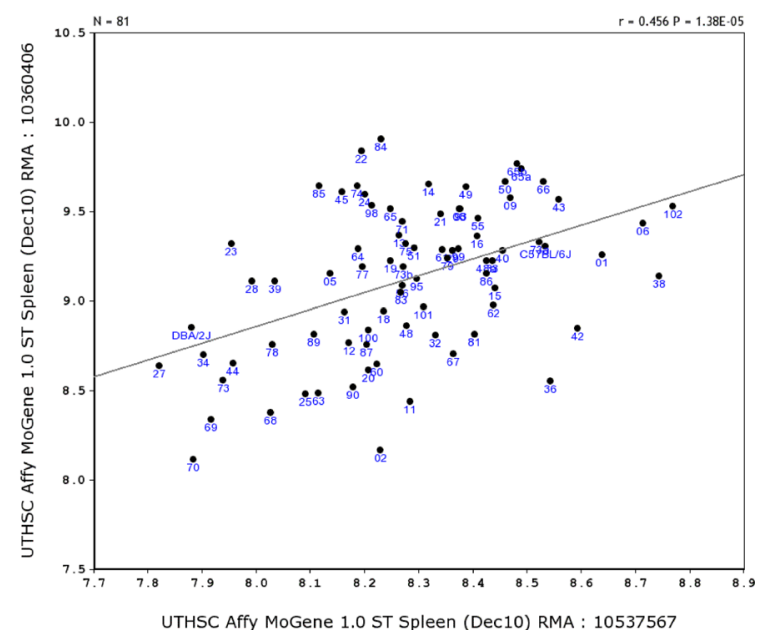

Fig. 6 Involvement of interferon-activatable protein and T-cell receptor beta chain genes in the resistance to SAD. a Gene expression levels of congenic strain in comparing with four other strains. Numbers on the left vertical bar are fold changes. Names of probes/genes are listed below the figure on horizontal axis. Four different strains are labeled with different colors. Expression levels of genes in congenic strain are down regulated in comparing with that of $\mathrm{BALB}^{-/-}$and $\mathrm{BALB} / \mathrm{c}$ but not significant difference to the other two strains, the ${ }^{-/-} \mathrm{DBA} / 1^{-/-}$and $\mathrm{DBA} / 1$. $\mathbf{b}$ Positive correlation of gene expression levels between Ifi203 (probe 10360391) and one probe for T-cell receptor beta, joining region (Tcrb-j) (Probe ID: 10537567) from the whole genome profiles of mouse spleen in BXD strains. Numbers on the left vertical bar are the relative expression level of Tcrb-j of an individual. Names listed below the figure on horizontal axis are the relative expression levels of Ifi203 of an individual. c Positive correlation of gene expression levels between Ifi205 (probe 103603406) and Tcrb-j (probe 10537567). Numbers on the left vertical bar are the relative expression level of Tcrb-j of an individual. Names listed below the figure on horizontal axis are the relative expression levels of Ifizo5 of an individual

possible factors that may lead to errors or variations of data, we have conducted real time qPCR to confirm the microarray data using Ifi203 as one of the key genes in this study. AS shown in Fig. 7, the expression level of Ifi203 in $\mathrm{BALB} / \mathrm{c}$ and $\mathrm{BALB} / \mathrm{c}-/$ - are high while in $\mathrm{DBA} / 1$ and DBA/1-/- are low. In BALB.D1-1, its expression level is the lowest among these strains and is much lower than that in BALB/C and BALB/c-/-. This data confirms that the decreased expression level of Ifi203 in BALB.D1-1 detected by microarray.

\section{Discussion}

Our multiple comparisons confirmed that the decreased expression levels of genes in T-cell receptor beta chain and Ifi cluster in the congenic strain are the major change that potentially leads to the increased resistance to SAD in the congenic strain in comparing to that of $\mathrm{BALB} / \mathrm{c}^{-/-}$. The expression levels of these genes are similar to that in DBA and DBA $/ 1^{-1-}$, which are resistant to the SAD. The expression of these genes is lower than that of $\mathrm{BALB} / \mathrm{c}$ and $\mathrm{BALB} / \mathrm{c}^{-/}$, which are susceptible to 


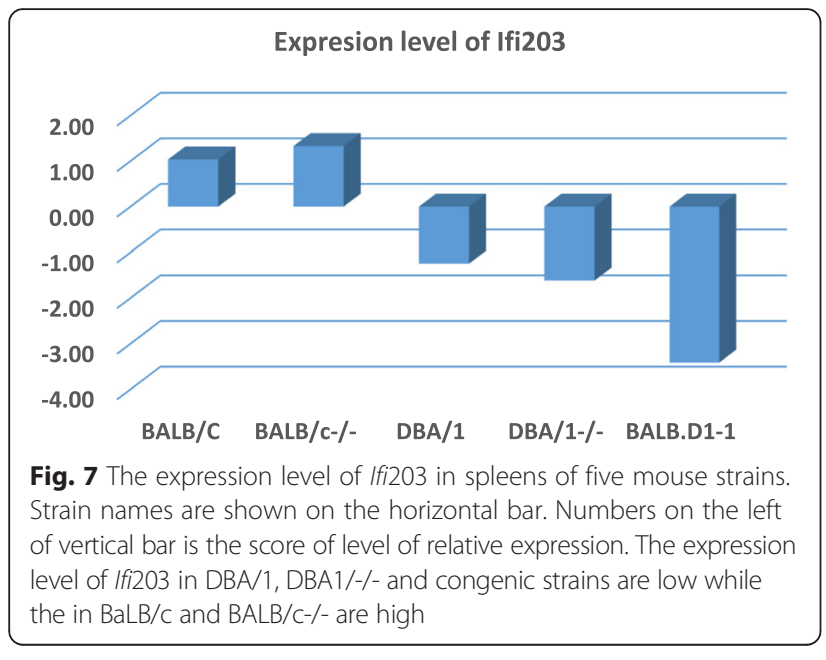

SAD. There are considerable number of genes that are differentially expressed between the congenic strain and three other parental strains, the DBA, ${ }^{-1} \mathrm{DBA} / 1^{-/}$and $\mathrm{BALB} / \mathrm{c}$ because of the different genomic background and mutation of Il1r. However, there is only one difference between the congenic strain and BALB $/ \mathrm{c}^{-/-}$. The difference is that in congenic mice, the genome region of QTL on chromosome 1 is replaced by the fragment from DBA while the entire remainder of the genome of $\mathrm{BALB} / \mathrm{c}^{-/-}$are from the BALB/c strain. The small number of candidate genes derived from our analyses pave the way to final determine the causal gene in the congenic strain.

Our preliminary data indicated the Ifi gene 200 cluster contains the favorite candidate gene for the SAD resistance in the congenic strain. Among differentially expressed genes between congenic and $\mathrm{BALB} / \mathrm{c}^{-1-}$, four genes in the Ifi gene 200 cluster are differentially expressed and are located in transferred genomic fragments in congenic strains. These genes are located in the transferred QTL genomic fragment on mouse chromosome 1, which is between D1Mit110 (167758517-167758653) and D1Mit209 (191493187-191493284). Our earlier analysis indicated that four candidate genes: Fcerlg, Fcgr3, Ifi202b, and Kmo are among the differentially expressed genes [29, 34]. Our current analysis indicates that the other three genes, Fcer1g, Fcgr3, and Kmo, were not differentially regulated in congenic strains compared to $\mathrm{BALB} / \mathrm{c}^{-/-}$that is susceptible to spontaneous arthritis. Interferon-activatable protein has been reported as an immune suppressor [36, 38-40]. For example, Ifi202 is known as an interferon-inducible lupus susceptibility gene $[36,38]$. Silencing of Ifi202b in tolerized CD8+ Ti cells abrogated the suppressive capacity of these cells. Recently the role of Ifi204 in the response to bacterial infection has been investigated [39]. It is expected that in the near future much broad function of Ifi family genes in the infection and immune innate immune responses will be identified [40]. Accordingly, it is very important to further investigate their candidacy.

The regulatory role of the T-cell receptor beta chain in the susceptibility of arthritis is well known [41-43]. Current information indicates that genes of T-cell receptor beta chain are not located in the transferred genomic fragment of the QTL region in the congenic strain. However, it is important to investigate if the genes between T-cell receptor beta chain family and Ifi family interact to down regulate SAD in the congenic strain.

In addition to the T-cell receptor beta chain and Ifi genes, we also detected the differential expression of Lefty1 in the QTL region. Early study indicated that Lefty1, Lefty2, and Nodal are expressed on the left side of developing mouse embryos and are implicated in L-R determination. Recently, because of the unique ability of Lefty to reroute the cell fate by making cells nonresponsive to diverse differentiation factors such as Nodal or other signals that require Egfcfc as a co-receptor, Lefty has become regarded as a determinant of cell stemness or differentiative events. There is no report for its role in arthritis, inflammation or infection. At present, we do not have enough information to explain its role in the congenic strains.

Our study was done with three replicates, each from female mice from each strain. The data may reflect a bias due to the use of female mice and may not represent that from male mice. Sex hormones have been shown to differentially regulate expression of Ifi202 [44]. However, all the strains are measured at the same age stge. Therefore, the differences at least reflect the genomic variation in the female mice between congenic and other strains. We used the spleen as the organ for the RNA extraction and whole genome gene profiling [28]. Although spleen as an organ important for immune, the phenotype of SAD in these mice were based on the joints. Therefore, we do not rule out that possibility that the data may not reflect what all happened in the joints. However, we believe that the molecular mechanism detected from this study is important to elucidate the causal gene for the congenic strain. Further study to directly link the candidate to the disease is necessary.

The "interferon-activatable protein" was labeled in the Illumina chip for a probe of LOC100040462. However, it lately has been validated as a transcription factor called $M n d a l$, which is also a member of Ifi family gene. Interestingly, Zhang et al. in their paper indicated that Mndal is absent from DBA/2j [45]. We assume that DBA/1, the strain we used in this study, is also absent. The increased level of expression level of Mndal in this case perhaps represents the Mndal gene from absent to present. However, the expression level from the DBA/1j genome detected by this probe is above the none-expression level (Absolute value over 118) while the expression level 
from the Balb/c genome is twice higher than that of the DBA/1j genome. As to its potential effect, it could be to interact with other genes in the If family or to affect the expression level of heat hsp105/110hDa and Igkc2-112, which are only two known genes which showed the decreased expression between the congenic strain and the most closest parental strain, DBA-/-. However, because of the differential expression level of other genes in Ifi family, whether Mndal affect the expression of hsp105/ $110 \mathrm{hDa}$ and Igkc2-112 need further study.

Our data indicated that many differentially expressed among those mouse strains are not associated with Il1-rn. This result is not completely unexpected. First of all, SAD occurs in the mouse strain that is lack of expression of Il1-rn. One would predict that the gene expression profile of such a mouse strain does not reflect the pathways that involves the Il1-rn. Secondly, the causal genes derived from the comparison between the congenic strain BALB.D1-1 and BALB/c-/- are under the genomic background of lack of Il1-rn. Thus, the causal genes are in fact acting when there is a lack of Il1-rn. It is important to notice that with standard protocol, CIA has been induced in DBA/1 and B6 [3-5]. Under standard protocol, CIA could not be induced in BALB/C mice without modification. The SAD in interleukin-1 (IL-1) receptor antagonist (IL-1rn) -deficient mice is dependent on non-MHC (major histocompatibility complex) genes in mice. It is well-known that Il1-rn has played an important role in the arthritis. The role of Ifi family genes in SAD may act in the population that is non-MHC and lack of role of Il1-rn. On the other hand, the lack of association in the gene expression does not rule out the protein-protein interaction. The protein products of these two gene families may be directly interact or binding to each other.

\section{Conclusions}

Our study demonstrated the association of expression levels of the Ifi gene 200 cluster to the susceptibility of mice to SAD. Our data also suggest that the regulation of SAD by the If $i$ gene 200 cluster is potentially through the regulation of genes of T-cell receptor beta chain.

\section{Additional files}

Additional file 1: Table S1. Down and up regulated genes comparing congenic strain to that in DBA wild type. List of names and DiffScores of changes of down or up regulated genes in congenic strain in comparison to that of DBA wild type. (XLSX $26 \mathrm{~kb}$ )

Additional file 2 Figure S1. Network of known arthritis relevant up and down regulated genes when congenics vs DBA/1 wild type. The 138 nodes in the graph below show the selected traits. Only nodes with edges are displayed. The 167 edges between the nodes, filtered from the 9453 total edges and drawn as lines, show Pearson correlation coefficients greater than 0.75 or less than -0.75 . The graph's canvas is 40.0 by $40.0 \mathrm{~cm}$, and the node labels are drawn with a 16.0 point font, and the edge labels are drawn with a 16.0 point font. Figure S2 Network of known arthritis relevant up and down regulated genes when congenics vs DBA ${ }^{-1}$. The 101 nodes in the graph below show the selected traits. Only nodes with edges are displayed. The 38 edges between the nodes, filtered from the 5050 total edges and drawn as lines, show Pearson correlation coefficients greater than 0.75 or less than -0.75 . The graph's canvas is 40.0 by $40.0 \mathrm{~cm}$, and the node labels are drawn with a 16.0 point font, and the edge labels are drawn with a 16.0 point font. Figure S3 Network of known arthritis relevant up and down regulated genes when congenics are compared to BALB/C wild type. The 92 nodes in the graph below show the selected traits. Only nodes with edges are displayed. The 40 edges between the nodes, filtered from the 4186 total edges and drawn as lines, show Pearson correlation coefficients greater than 0.75 or less than -0.75 . The graph's canvas is 40.0 by $40.0 \mathrm{~cm}$, and the node labels are drawn with a 16.0 point font, and the edge labels are drawn with a 16.0 point font. (DOCX 71 kb)

Additional file 3: Table S2. Down and up regulated genes comparing congenic strain to that of DBA $111 \mathrm{rn}$ mutation. List of names and DiffScores of changes of down or up regulated genes in congenic strain in comparison to that of DBA $111 \mathrm{rn}$ mutation. (XLSX $21 \mathrm{~kb}$ )

Additional file 4: Table S3. Down and up regulated genes comparing congenic stain to the Balb/c wild type. List of names and DiffScores of changes of down or up regulated genes in congenic strain in comparison to that of Balb/c wild type. (XLSX $10 \mathrm{~kb}$ )

\section{Abbreviations}

CD4, cluster of differentiation 4; CIA, collagen-induced arthritis; Ifi, interferonactivatable protein; II-1ra, IL-1 receptor antagonist protein; PCR, polymerase chain reaction; QTL, quantitative trait loci; $S A D$, spontaneous arthritis disease; Tcrb, T-cell receptor beta chain

\section{Acknowledgements}

This work was partially supported funding from The first hospital of Qiqihare City and merit grants (I01 BX000671 to WG, 1IPIBX001607 to JS) from the Department of Veterans Affairs and the Veterans Administration Medical Center in Memphis, TN, USA and National Natural Science Foundation of China (Project 81372996 to YJ; Project 81171679 to YHC), P.R. China.

\section{Availability of supporting data}

The data set(s) supporting the results of this article are included in the supplementary data of this manuscript.

\section{Authors' contributions}

YJ carried out the molecular genetic studies, participated in the microarray experiments, analyzed the data, and participating in drafting of the manuscript. $\mathrm{XL}$ carried out the animal breeding and genotyping, and revised the manuscript. YH carried out the animal breeding and revised the manuscript. ND carried out the animal breeding. YM participated in the microarray studies. HC participated in the design of the study, and helped to revise the manuscript. KH conceived of the study, and participated in its design and helped to draft the manuscript. AK conceived of the study, and participated in its design and helped to draft the manuscript. JS conceived of the study, and participated in its design and helped to draft the manuscript. WG conceived of the study, participated in its design, analyzed the data, and helped to finalize the manuscript. All authors read and approved the final manuscript.

\section{Competing interests}

The authors declare no competing interest.

\section{Consent for publication}

Not applicable

\section{Ethics approval and consent to participate}

This study did not involve human subjects or samples.

All mice have been maintained in the animal facility of the Department of Veterans Affairs Medical Center, Memphis. Experimental animal procedures and mouse husbandry were performed in accordance with the National Institutes of Health's Guide for the Care and Use of Laboratory Animals and approved by the VAMC Institutional Animal Care and Use Committee, Protocol \#: 334310. 


\section{Author details}

'Mudanjiang Medical College, Mudanjiang, HeilongJiang 157001, People's Republic of China. ${ }^{2}$ Department of Orthopaedic Surgery and Biomedical Engineering, University of Tennessee Health Science Center (UTHSC), Memphis, TN 38163, USA. ${ }^{3}$ Center of integrative research, The first Hospital of Qiqihaer City, 30 Gongyuan Road, Longsha District, Qiqihaer, Heilongjiang 161005, People's Republic of China. ${ }^{4}$ Institute of Kaschin-beck Disease, Center for Endemic Disease Control, Chinese Center for Disease Control and Prevention, Harbin Medical University, Harbin 150081, China. ${ }^{5}$ Key Laboratory of Etiologic Epidemiology, Education Bureau of Heilongjiang Province \& Ministry of Health (23618104), Harbin 150081, China. 'Department of Medicine, University of Tennessee Health Science Center, Memphis, TN 38163, USA. 'Research Service, Veterans Affairs Medical Center, 1030 Jefferson Avenue, Memphis, TN 38104, USA.

Received: 31 March 2016 Accepted: 12 July 2016

Published online: 02 August 2016

\section{References}

1. Wooley PH, Luthra HS, Stuart JM, David CS. Type II collagen-induced arthritis in mice. I. Major histocompatibility complex (I region) linkage and antibody correlates. J Exp Med. 1981:154(3):688-700.

2. Campbell EC, Antoniou AN, Powis SJ. The multi-faceted nature of HLA class I dimer molecules. Immunology. 2012;136(4):380-4. doi:10.1111/j.1365-2567. 2012.03593.x. Review.

3. Brand DD, Latham KA, Rosloniec EF. Collagen-induced arthritis. Nat Protoc. 2007;2(5):1269-75.

4. Pietrosimone KM, Jin M, Poston B, Liu P. Collagen-Induced Arthritis: A model for Murine Autoimmune Arthritis. Bio Protoc. 2015;5(20). pii: e1626.

5. Griffiths MM, Remmers EF. Genetic analysis of collagen-induced arthritis in rats: a polygenic model for rheumatoid arthritis predicts a common framework of cross-species inflammatory/autoimmune disease loci. Immunol Rev. 2001;184 172-83. Review.

6. Adarichev VA, Valdez JC, Bardos T, Finnegan A, Mikecz K, Glant TT. Combined autoimmune models of arthritis reveal shared and independent qualitative (binary) and quantitative trait loci. J Immunol. 2003;170:2283-92

7. Holmdahl R, Lorentzen JC, Lu S, Olofsson P, Wester L, Holmberg J, Pettersson U. Arthritis induced in rats with nonimmunogenic adjuvants as models for rheumatoid arthritis. Immunol Rev. 2001;184:184-202.

8. Park KS, Park MJ, Cho ML, Kwok SK, Ju JH, Ko HJ, Park SH, Kim HY. Type II collagen oral tolerance; mechanism and role in collagen-induced arthritis and rheumatoid arthritis. Mod Rheumatol. 2009;19(6):581-9.

9. van Heemst J, van der Woude D, Huizinga TW, Toes RE. HLA and rheumatoid arthritis: how do they connect? Ann Med. 2014;46(5):304-10.

10. Rowley MJ, Nandakumar KS, Holmdahl R. The role of collagen antibodies in mediating arthritis. Mod Rheumatol. 2008;18(5):429-41.

11. Schurgers E, Billiau A, Matthys P. Collagen-induced arthritis as an animal model for rheumatoid arthritis: focus on interferon- $\gamma$. J Interferon Cytokine Res. 2011:31(12):917-26.

12. Andersson A, Stubelius A, Karlsson MN, Engdahl C, Erlandsson M, Grahnemo L, Lagerquist MK, Islander U. Estrogen regulates T helper 17 phenotype and localization in experimental autoimmune arthritis. Arthritis Res Ther. 2015;17:32.

13. Kato G, Shimizu Y, Arai Y, Suzuki N, Sugamori Y, Maeda M, Takahashi M, Tamura Y, Wakabayashi N, Murali R, Ono T, Ohya K, Mise-Omata S, Aoki K. The inhibitory effects of a RANKL-binding peptide on articular and periarticular bone loss in a murine model of collagen-induced arthritis: a bone histomorphometric study. Arthritis Res Ther. 2015;17:251.

14. Van Doornum S, Bohensky M, Tacey MA, Brand CA, Sundararajan V, Wicks IP. Increased 30-day and 1-year mortality rates and lower coronary revascularisation rates following acute myocardial infarction in patients with autoimmune rheumatic disease. Arthritis Res Ther. 2015;17:38

15. Abdollahi-Roodsaz S, Joosten LA, Koenders MI, Devesa I, Roelofs MF, Radstake TR, Heuvelmans-Jacobs M, Akira S, Nicklin MJ, Ribeiro-Dias F, van den Berg WB. Stimulation of TLR2 and TLR4 differentially skews the balance of T cells in a mouse model of arthritis. J Clin Invest. 2008;118(1):205-16.

16. Nakae S, Saijo S, Horai R, Sudo K, Mori S, Iwakura Y. IL-17 production from activated $T$ cells is required for the spontaneous development of destructive arthritis in mice deficient in IL-1 receptor antagonist. Proc Natl Acad Sci U S A. 2003;100(10):5986-90.
17. Wade CM, Kulbokas 3rd EJ, Kirby AW, Zody MC, Mullikin JC, Lander ES, Lindblad-Toh K, Daly MJ. The mosaic structure of variation in the laboratory mouse genome. Nature. 2002;420(6915):574-8.

18. Pasold J, Osterberg A, Peters K, Taipaleenmäki H, Säämänen AM, Vollmar B, Müller-Hilke B. Reduced expression of Sfrp1 during chondrogenesis and in articular chondrocytes correlates with osteoarthritis in STR/ort mice. Exp Cell Res. 2013;319(5):649-59.

19. van Dongen J, Jansen $R$, Smit D, Hottenga JJ, Mbarek H, Willemsen G, Kluft C, AAGC Collaborators, Penninx BW, Ferreira MA, Boomsma DI, de Geus EJ. The contribution of the functional IL6R polymorphism rs2228145, eQTLs and other genome-wide SNPs to the heritability of plasma sIL-6R levels. Behav Genet. 2014;44(4):368-82.

20. De Franco M, Peters LC, Correa MA, Galvan A, Canhamero T, Borrego A, Jensen JR, Gonçalves J, Cabrera WH, Starobinas N, Ribeiro OG, Dragani T, Ibañez OM. Pristane-induced arthritis loci interact with the Slc11al gene to determine susceptibility in mice selected for high inflammation. PLoS One. 2014;9(2):e88302.

21. Jesus AA, Goldbach-Mansky R. IL-1 blockade in autoinflammatory syndromes. Annu Rev Med. 2014;65:223-44. doi:10.1146/annurev-med-061512-150641.

22. Besenyei T, Kadar A, Tryniszewska B, Kurko J, Rauch TA, Glant TT, Mikecz K, Szekanecz Z. Non-MHC risk alleles in rheumatoid arthritis and in the syntenic chromosome regions of corresponding animal models. Clin Dev Immunol. 2012;2012:284751.

23. Nandakumar KS, Holmdahl R. A genetic contamination in MHC-congenic mouse strains reveals a locus on chromosome 10 that determines autoimmunity and arthritis susceptibility. Eur J Immunol. 2005;35(4):1275-82.

24. Atchley WR, Fitch WM. Gene trees and the origins of inbred strains of mice. Science. 1991;254(5031):554-8.

25. Zhou F, He X, Iwakura Y, Horai R, Stuart JM. Arthritis in mice that are deficient in interleukin-1 receptor antagonist is dependent on genetic background. Arthritis Rheum. 2005:52:3731-8.

26. Jiao Y, Jiao F, Yan J, Xiong Q, Shriner D, Hasty K, Stuart J, Gu W. Identifying a major locus that regulates spontaneous arthritis in IL-1ra-deficient mice and analysis of potential candidates. Genet Res (Camb). 2011;93(2):95-103.

27. Cao Y, Zhang J, Jiao Y, Yan J, Jiao F, Liu X, Williams RW, Hasty KA, Stuart JM, Gu W. Genomic dissection and prioritizing of candidate genes of QTL for regulating spontaneous arthritis on chromosome 1 in mice deficient for interleukin-1 receptor antagonist. J Genet. 2012;91(2):119-28.

28. Yan J, Jiao Y, Chen H, Jiao F, Hasty KA, Stuart JM, Gu W. Dual effects of IL-1 overactivity on the immune system in a mouse model of arthritis due to deficiency of IL-1 receptor antagonist. J Genet Genomics. 2013;40(2):83-91.

29. Cao Y, Liu X, Deng N, Jiao Y, Ma Y, Hasty KA, Stuart JM, Gu W. Congenic mice provide evidence for a genetic locus that modulates spontaneous arthritis caused by deficiency of IL-1RA. PLoS One. 2013;8(6):e68158.

30. Gu W, Li X, Lau KH, Edderkaoui B, Donahae LR, Rosen CJ, Beamer WG, Shultz KL, Srivastava A, Mohan S, Baylink DJ. Gene expression between a congenic strain that contains a quantitative trait locus of high bone density from CAST/ EiJ and its wild-type strain C57BL/6 J. Funct Integr Genomics. 2002;1 (6):375-86.

31. Yu H, Lu C, Tan MT, Moudgil KD. Comparative antigen-induced gene expression profiles unveil novel aspects of susceptibility/resistance to adjuvant arthritis in rats. Mol Immunol. 2013;56(4):531-9.

32. Wang $L$, Lu W, Zhang $L$, Huang $Y$, Scheib R, Liu X, Myers $L$, Lu L, Farber CR, Liu G, Wang CY, Deng H, Williams RW, Wang Y, Gu W, Jiao Y. Trps1 differentially modulates the bone mineral density between male and female mice and its polymorphism associates with BMD differently between women and men. PLoS One. 2014;9(1):e84485.

33. Jiao Y, Chen H, Yan J, Wang L, Huang Y, Liu X, Williams RW, Lu L, Wang Y, Gu W. Genome-wide gene expression profiles in antioxidant pathways and their potential sex differences and connections to vitamin C in mice. Int J Mol Sci. 2013;14(5):10042-62

34. Cao Y, Jiao Y, Wang L, Huang Y, Postlethwaite A, Stuart J, Kang A, Williams RW, Gu W. Anakinra as an interleukin 1 receptor antagonist, complicated genetics and molecular impacts-from the point of view of mouse genomics. Int Immunopharmacol. 2012;13(1):28-36.

35. Wang L, Jiao Y, Sun S, Jarrett HW, Sun D, Gu W. Gene network of a phosphoglycerate mutase in muscle wasting in mice. Cell Biol Int. 2015; 39(6):666-77.

36. Dinesh R, Hahn BH, La Cava A, Singh RP. Interferon-inducible gene 202b controls CD8+ T cell-mediated suppression in anti-DNA Ig peptide-treated $(N Z B \times N Z W)$ F1 lupus mice. Genes Immun. 2011;12:360-9.

37. Xiong Q, Qiu Y, Gu W. PGMapper: a web-based tool linking phenotype to genes. Bioinformatics. 2008;24(7):1011-3. 
38. Chen J, Panchanathan R, Choubey D. Stimulation of T cells up-regulates expression of IfizO2, an interferon-inducible lupus susceptibility gene, through activation of JNKJc-Jun pathway. Immunol Lett. 2008;1 18(1):13-20.

39. Storek KM, Gertsvolf NA, Ohlson MB, Monack DM. CGAS and Ifizo4

Cooperate To Produce Type I IFNs in Response to Francisella Infection. J Immunol. 2015;194(7):3236-45.

40. Zhao H, Gonzalezgugel E, Cheng L, Richbourgh B, Nie L, Liu C. The roles of interferon-inducible p200 family members IFI16 and p204 in innate immune responses, cell differentiation and proliferation. Genes Dis. 2015;2(1):46-56.

41. Jenkins MK, Melvold RW, Miller SD. Isolation and characterization of an I-Arestricted T cell clone with dual speclficity for poly(Glu60Ala30Tyr10) (GAT) and Mlsa, dl. J Immunol. 1984;133(2):616-22.

42. Merky P, Batsalova T, Bockermann R, Dzhambazov B, Sehnert B, Burkhardt H, Bäcklund J. Visualization and phenotyping of proinflammatory antigenspeclfic T cells during collagen-induced arthritis in a mouse with a fixed collagen type Il-speclfic transgenic T-cell receptor $\beta$-chain. Arthritis Res Ther. 2010;12(4):R155

43. Berlo SE, van Kooten PJ, Ten Brink CB, Hauet-Broere F, Oosterwegel MA, Glant TT, Van Eden W, Broeren CP. Naive transgenic T cells expressing cartilage proteoglycan-spec/fic TCR induce arthritis upon in vivo activation. J Autoimmun. 2005;25(3):172-80.

44. Panchanathan R, Shen H, Bupp MG, Gould KA, Choubey D. Female and male sex hormones differentially regulate expression of IfizO2, an interferoninducible lupus susceptibility gene within the Nba2 interval. J Immunol. 2009;183(11):7031-8.

45. Zhang K, Kagan D, DuBois W, Robinson R, Bliskovsky V, Vass WC, Zhang S, Mock BA. Mndal, a new interferon-inducible family member, is highly polymorphic, suppresses cell growth, and may modify plasmacytoma susceptibility. Blood. 2009;114(14):2952-60. doi:10.1182/blood-2009-01198812. Epub 2009 Aug 4

\section{Submit your next manuscript to BioMed Central and we will help you at every step:}

- We accept pre-submission inquiries

- Our selector tool helps you to find the most relevant journal

- We provide round the clock customer support

- Convenient online submission

- Thorough peer review

- Inclusion in PubMed and all major indexing services

- Maximum visibility for your research

Submit your manuscript at www.biomedcentral.com/submit

) Biomed Central 